\title{
Epidemiological investigation of community-acquired Staphylococcus aureus infection
}

\author{
W. Jiang, Z. Zhou, K. Zhang and Y. Yu \\ Department of Clinical Laboratory, The First Affiliated Hospital of the General \\ Hospital of the People's Liberation Army, Beijing, China \\ Corresponding author: Y. Yu \\ E-mail: weijiangcn@163.com
}

Genet. Mol. Res. 12 (4): 6923-6930 (2013)

Received August 2, 2013

Accepted November 27, 2013

Published December 19, 2013

DOI http://dx.doi.org/10.4238/2013.December.19.11

\begin{abstract}
The current study aimed to reveal the pathogenic and spreading mechanisms of community-acquired Staphylococcus aureus (CA-SA) by analyzing its prevalence, drug resistance, virulence, and pathogenic factors. Historical information regarding CA skin and soft tissue infections were collected and disease characteristics were analyzed. Isolated CA-SA strains were subjected to antibiotic sensitivity tests using the agar dilution method, Staphylococcus cassette chromosome mec (SCCmec) tests, multilocus sequence typing (MLST), and staphylococcal protein A (SPA) and toxin gene screening. A total of 55 skin and soft tissue infections were investigated, and 12 strains of SA bacteria were isolated, which were all CA methicillin-susceptible SA (CA-MSSA). The antibiotic sensitivity tests showed that CA-MSSA was susceptible to all antibacterials with the exception of high resistance to erythromycin, clindamycin, tetracycline, gentamicin, and levofloxacin (ranging from 8.3 to $50 \%$ ). The toxin detection results showed that the Panton-Valentine leukocidin (PVL) positive rate in CA-MSSA was $33.3 \%$, the enterotoxin positive rate was $25 \%$, and the toxic shock
\end{abstract}


syndrome toxin-1 (TSSL) positive rate was $8.3 \%$. No Staphylococcus enterotoxin H or Staphylococcus exfoliati was detected. MLST and SPA typing showed that the clones of CA-MSSA included ST5-t002 (2 strains), ST22-t309 (2 strains), ST398-t034, ST15-t5864, ST7-t091, ST25-t078, ST30-t318, ST121-t1425, ST800-t1425, and ST630-t377. No methicillin-resistant SA (MRSA) strains were detected in this study. CA-MSSA strains have high drug susceptibility and diverse genetic backgrounds. The coexistence of multiple toxins may provide a survival advantage in community dissemination for CA-MSSA.

Key words: Skin and soft tissue infection; Multilocus sequence typing; Community-acquired Staphylococcus aureus; Staphylococcal protein A; Panton-Valentine leukocidin

\section{INTRODUCTION}

Staphylococcus aureus (SA) is a common pathogenic bacterium that leads to hospitalacquired (HA) and community-acquired (CA) infections mainly including skin and soft tissue infections (SSTIs) (DeLeo et al., 2010; Mithoe et al., 2012; Zhao et al., 2012) and severe fatal diseases such as endocarditis, necrotic pneumonia, severe bacteremia, and toxic shock syndrome. CA-SA is the primary pathogenic bacterium of SSTIs treated in emergency departments (Chen et al., 2010; DeLeo et al., 2010; Mithoe et al., 2012; Zhao et al., 2012). Although HA-SA has been extensively investigated (Liu et al., 2009; Chen et al., 2010; Wu et al., 2010; Mithoe et al., 2012), studies on CA-SA, particularly CA-SA-caused SSTIs in adults, are rare (Wu et al., 2010; Zhao et al., 2012). Some researchers have hypothesized that methicillinresistant SA (MRSA) is caused by the transfer of Staphylococcus cassette chromosome mec (SCCmec) to methicillin-susceptible SA (MSSA) (Lindsay and Holden, 2006; Pandian et al., 2011). Therefore, investigating the molecular structure and genetic makeup of CA-MSSA is of great significance for understanding CA-MRSA formation. In addition, virulence factors play an important role in CA-SA infections (Kaltsas et al., 2011; Khokhlova et al., 2012), such as Panton-Valentine leukocytic toxin (PVL), toxic shock syndrome toxin-1 (TSST-1), staphylococcal enterotoxin C (SEC), staphylococcal enterotoxin H (SEH), staphylococcal exfoliative toxins (ET), and so on.

In this study, we summarized the clinical features, epidemiological distribution, molecular characteristics, and drug resistance of 55 CA-SA-caused SSTIs treated at our hospital. The aims of the study were to elucidate the incidence, drug resistance phenotypes, risk factors, toxigene carrying rate, and molecular characteristics of CA-MSSA, and thus to provide laboratory data for the treatment of CA-MSSA-caused infections.

\section{MATERIAL AND METHODS}

\section{Case selection and strain collection}

Patients with CA purulent SSTI that received treatment at the dermatology, outpatient, and emergency departments of our hospital were enrolled in the study. Strains were primarily 
obtained through isolation from the selected cases between January 2009 and August 2010. The quality-controlled strains were SA ATCC29213 and Enterococcus faecalis ATCC29212. This study was conducted in accordance with the declaration of Helsinki and with approval from the Ethics Committee of the First Affiliated Hospital of the Chinese People's Liberation Army General Hospital. Written informed consent was obtained from all participants.

\section{Strain identification}

Preliminary identification was performed based on bacterial morphology, Gram staining, hemolysis, and catalase tests. Then, Slidex Staph Plus latex agglutination was performed for rapid detection; agglutinating bacteria that could be visually observed within 30 s were determined to be SA. Questionable bacteria were further identified using the API and VITEK systems.

\section{Antibacterial sensitivity test}

The minimal inhibitory concentrations (MICs) of 18 antibacterials for 12 SA strains were determined using agar dilution based on the Clinical and Laboratory Standards Institute M100-S20 2010 criteria proposed by the National Committee for Clinical Laboratory Standards (Clinical and Laboratory Standards Institute, 2010). The antibacterials for the determination of sensitivity, mesomerisms, and drug resistance included oxacillin (Sigma Chemical; St. Louis, MO, USA), cefoxitin (FOX; Sigma Chemical), teicoplanin (Sanofiaventis; Schiltigheim, France), vancomycin (Eli Lilly; Indianapolis, IN, USA), erythromycin (ERY; Sigma Chemical), clindamycin (CLI; Sigma Chemical), tetracycline (TCY), chloramphenicol (Sigma Chemical), gentamicin (GEN; Sigma Chemical), sulfamethoxazole + trimethoprim (Sigma Chemical), rifampicin (Sigma Chemical), levofloxacin (LVX; Daiichi Pharmaceutical; Tokyo, Japan), tigecycline (Pfizer Pharmaceutical; New York, NY, USA), linezolid (Pfizer), cephazolin (Sigma Chemical), cefuroxime (Sigma Chemical), ceftriaxone (Sigma Chemical), and mupirocin (Sigma Chemical). The breakpoint of tigecycline was that proposed by the American Food and Drug Administration $(\mathrm{S} \leq 0.5 \mu \mathrm{g} / \mathrm{mL})$.

\section{MRSA detection}

The isolated SA strains were detected using the oxacillin MIC method and mecA and femB duplex polymerase chain reactions (PCRs) (Sun et al., 2012; Zhao et al., 2012). The duplex PCR conditions consisted of $94^{\circ} \mathrm{C}$ for 4 min, 30 cycles of $94^{\circ} \mathrm{C}$ for $45 \mathrm{~s}, 50^{\circ} \mathrm{C}$ for 45 $\mathrm{s}$, and $72^{\circ} \mathrm{C}$ for $60 \mathrm{~s}$, and a final step at $72^{\circ} \mathrm{C}$ for $2 \mathrm{~min}$. The electrophoresis strips of mecA and femB were respectively located at 310 and $651 \mathrm{bp}$. If both strips showed positive results, the strain was determined to be MRSA. If only mecA was positive, the strain was judged as methicillin-resistant coagulase-negative staphylococci (MRSCN). If only femB was positive, the strain was judged as MSSA.

\section{CA-SA molecular typing}

SCCmecA typing, staphylococcal protein A (SPA) typing, and multilocus sequence typing (MLST) were performed using PCR according to the literature (Rodríguez-Baño et al., 
2009; Escobar et al., 2012; Sun et al., 2012; Zhao et al., 2012). The seven housekeeping genes for MLST amplification were arc (673 bp), aroe (568 bp), glpf (635 bp), gmk (548 bp), pta (584 bp), tpi (512 bp), and yqil (654 bp). Strain MLST was determined, and the genetic relationships among different primary clones were analyzed according to the Allelic Profiles software. The primer sequences for SPA typing were: 5'-TAAAGACGATCCTTCGGYGAGC-3' (spal13f) and 5'-CAGCAGTAGTGCCGTTTGCTT-3' (spa1514f). The PCR conditions consisted of $95^{\circ} \mathrm{C}$ for $5 \mathrm{~min}, 30$ cycles of $95^{\circ} \mathrm{C}$ for $45 \mathrm{~s}, 60^{\circ} \mathrm{C}$ for $45 \mathrm{~s}$, and $72^{\circ} \mathrm{C}$ for $90 \mathrm{~s}$, and a final step at $72^{\circ} \mathrm{C}$ for $10 \mathrm{~min}$. A single strip between 300 and $400 \mathrm{bp}$ was considered to be positive. Forward sequencing was primarily performed using the spall $3 \mathrm{f}$ primer. The obtained $s p a$ sequencing outcomes were compared with those in the typing database (http://spa.ridom.de/ repeats.shtml).

\section{Virulence-related gene detection}

The virulence-related genes encoding PVL, TSST-1, SEC, SEH, and ET were amplified using PCR based on methods provided in the literature (Chen et al., 2009; Kaltsas et al., 2011; Khokhlova et al., 2012; Zhao et al., 2012). The target genes were 433 bp for PVL, 350 bp for TSST-1, 257 bp for SEC, 375 bp for SEH, and 119 bp for ET.

\section{Statistical analysis}

Drug susceptibility data were analyzed for drug resistance patterns using the WHONET-5.6 software and the interpretation of breakpoints was based on the guidelines of the Clinical and Laboratory Standards Institute. Data batch processing and integration were performed using the Microsoft Office and Filemaker database softwares.

\section{RESULTS}

\section{Clinical and microbiological characteristics of SSTIs}

A total of 55 SSTIs were investigated in this study. Among the recruited patients, 10 were male $(62.5 \%)$. The patients ranged in age from 19 to 62 years with an average age of $32.2 \pm 11.6$ years. Among the infections, there were 10 skin infections, 6 phlegmons, 5 festers, 5 furuncles, 8 folliculitises, 8 Tinea skin infections, 6 abscesses, and 7 other SSTIs. A total of 12 SA strains, 27 coagulase-negative Staphylococcus strains, and 15 other pathogenic strains were isolated. All of the strains were verified and confirmed using the API 20E or VITEK2 Compact automatic system.

\section{CA-SA drug susceptibility patterns and duplex PCR outcomes}

No MRSA was observed among the 12 strains of SA (FOX $>8 \mu \mathrm{g} / \mathrm{mL}$ ). CA-SA showed high resistance rates to ERY, CLI, TCY, GEN, and LVX $(50,25,25,8.3$, and 8.3\%, respectively); it showed susceptibility to the remaining 13 drugs tested $(100 \%)$. The results of CA-SA drug susceptibility are summarized in Table 1. Duplex PCR only showed positive femB results. Thus, CA-MSSA was defined. 


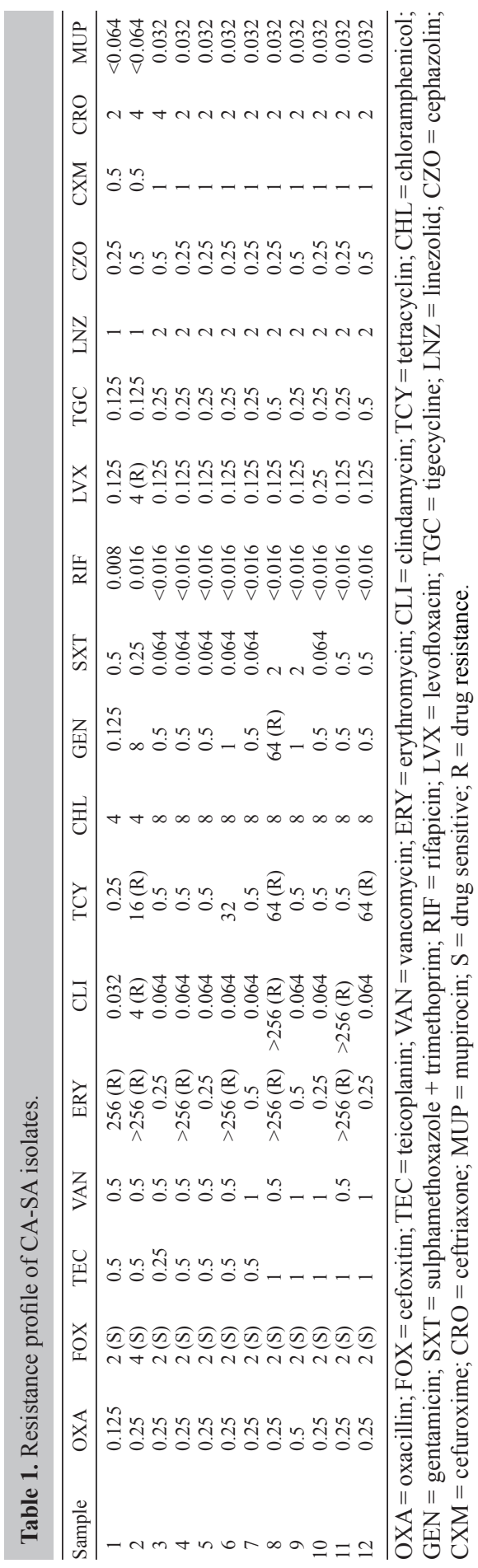




\section{MLST and SPA typing of CA-MSSA}

A total of 10 MLST-based types and 8 SPA-based types were confirmed in CA-MSSA, which belonged to 9 types of clonal complexes. The clones included ST5-t002-CC5 (2 strains), ST22-t309-CC22 (2 strains), ST398-t034-CC398 (1 strain), ST15-t5864-CC15 (1 strain), ST7-t091-CC7 (1 strain), ST25-t078-CC25 (1 strain), ST30-t318-CC30 (1 strain), ST121t1425-CC121 (1 strain), ST800-t1425-CC121 (1 strain), and ST630-t377-CC8 (1 strain). The distribution of MLST- and SPA-based CA-MSSA types are shown in Figure 1 and Table 2.

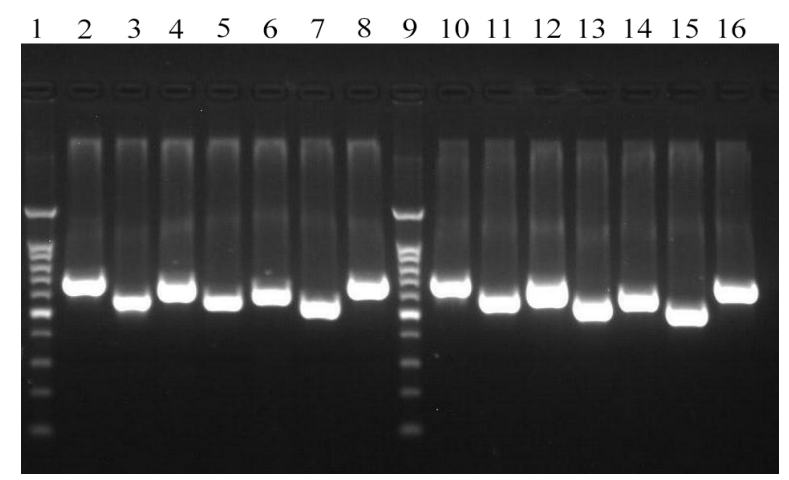

Figure 1. Results of PCR from two clinical cases of culture-positive MSSA bacteria. The PCR was conducted as described in the text and products were electrophoresed on $1 \%$ agarose-TAE gel. PCR results appear in duplicate (lanes 2 to 8 and 10 to 16). Lanes 1 and 9: 100-bp DNA ladder; lanes 2 to 8 and lanes 10 to 16: PCR for the arc, aroe, glpf, gmk, pta, tpi, and yqil genes, respectively. The sizes of the PCR amplicons are: 673 bp for arc, $568 \mathrm{bp}$ for aroe, $635 \mathrm{bp}$ for $\mathrm{glpf}, 548 \mathrm{bp}$ for $g m k, 584 \mathrm{bp}$ for $p t a, 512 \mathrm{bp}$ for tpi, and $654 \mathrm{bp}$ for yqil.

Table 2. Molecular characteristics of 12 isolated CA-MSSA strains.

\begin{tabular}{|c|c|c|c|c|c|c|c|c|c|c|}
\hline Sample & Identification & SCCmec & SPA & MLST & Clone complex & $p v l$ & $\mathrm{sec}$ & seh & tsst-1 & et \\
\hline 1 & sau & MSSA & $\mathrm{t} 318$ & 30 & $\mathrm{CC} 30$ & $\mathrm{P}$ & $\mathrm{P}$ & $\mathrm{N}$ & $\mathrm{P}$ & $\mathrm{N}$ \\
\hline 2 & sau & MSSA & t034 & 398 & CC398 & $\mathrm{N}$ & $\mathrm{N}$ & $\mathrm{N}$ & $\mathrm{N}$ & $\mathrm{N}$ \\
\hline 3 & sau & MSSA & t377 & 630 & $\mathrm{CC} 8$ & $\mathrm{~N}$ & $\mathrm{~N}$ & $\mathrm{~N}$ & $\mathrm{~N}$ & $\mathrm{~N}$ \\
\hline 4 & sau & MSSA & t309 & 22 & $\mathrm{CC} 22$ & $\mathrm{P}$ & $\mathrm{N}$ & $\mathrm{N}$ & $\mathrm{N}$ & $\mathrm{N}$ \\
\hline 5 & sau & MSSA & t309 & 22 & $\mathrm{CC} 22$ & $\mathrm{P}$ & $\mathrm{N}$ & $\mathrm{N}$ & $\mathrm{N}$ & $\mathrm{N}$ \\
\hline 6 & sau & MSSA & t091 & 7 & $\mathrm{CC} 7$ & $\mathrm{~N}$ & $\mathrm{~N}$ & $\mathrm{~N}$ & $\mathrm{~N}$ & $\mathrm{~N}$ \\
\hline 7 & sau & MSSA & t002 & 5 & CC5 & $\mathrm{N}$ & $\mathrm{N}$ & $\mathrm{N}$ & $\mathrm{N}$ & $\mathrm{N}$ \\
\hline 8 & sau & MSSA & $\mathrm{t} 002$ & 5 & CC5 & $\mathrm{N}$ & $\mathrm{P}$ & $\mathrm{N}$ & $\mathrm{N}$ & $\mathrm{N}$ \\
\hline 9 & sau & MSSA & $\mathrm{t} 1425$ & 121 & CC121 & $\mathrm{N}$ & $\mathrm{N}$ & $\mathrm{N}$ & $\mathrm{N}$ & $\mathrm{N}$ \\
\hline 10 & sau & MSSA & t5864 & 15 & $\mathrm{CC} 15$ & $\mathrm{~N}$ & $\mathrm{~N}$ & $\mathrm{~N}$ & $\mathrm{~N}$ & $\mathrm{~N}$ \\
\hline 11 & sau & MSSA & $\mathrm{t} 1425$ & 800 & CC121 & $\mathrm{N}$ & $\mathrm{N}$ & $\mathrm{N}$ & $\mathrm{N}$ & $\mathrm{N}$ \\
\hline 12 & sau & MSSA & t078 & 25 & $\mathrm{CC} 25$ & $\mathrm{P}$ & $\mathrm{P}$ & $\mathrm{N}$ & $\mathrm{N}$ & $\mathrm{N}$ \\
\hline
\end{tabular}

$\mathrm{P}=$ positive; $\mathrm{N}=$ negative.

\section{Virulence genes}

The $p v l$ gene was detected in 4 strains of CA-MSSA (33.3\%), the sec gene was found in 3 strains (25\%), and the tsst-1 gene was found in one strain (8.3\%). The $p v l$, sec, and tsst-1 genes were found coexisting in one strain, and another strain showed the coexistence of the $p v l$ and sec genes (Table 2). 


\section{DISCUSSION}

This study did not show CA-MRSA strains of the CA-SA isolated from adult SSTI patients at our hospital. All 12 of the CA-SA strains were subjected to CA-MSSA. The drug susceptibility tests showed that CA-MSSA displayed $100 \%$ susceptibility to all of the antibacterials tested, with the exception of high resistance rates to ERY, CLI, TCY, GEN, and LVX (ranging from 8.3 to $50 \%$ ). These findings suggest that antibiotics in the community evaluated may not be great threats to strain survival.

Furthermore, clones identical to HA-MSSA were observed in the 12 CA-MSSA strains investigated: ST5 (2 strains), ST398 (1 strain), and ST7 (1 strain). This finding indicates that the three clone strains are all correlated with CA and HA infections (Chen et al., 2010), which generally agrees with results reported previously (Du et al., 2011; Chen et al., 2012). ST398 is increasing annually in MRSA (Schijffelen et al., 2010; Smith and Pearson, 2011). Complete genome sequencing has shown that the ST398 strain lacks virulence factors such as enterotoxin (Schijffelen et al., 2010), which was confirmed in this study.

PVL-positive SA is often correlated with SSTIs, particularly phlegmons, abscesses, and furuncles. This study showed that the $p v l$ gene was present in $33.3 \%$ of the CA-MSSA strains, which was higher than the PVL-positive rates reported in children (4.2-6\%) (Huang and Chen, 2011; Kini et al., 2013; Pardo et al., 2013) and in other studies of HA strains (Chen et al., 2009; Kawaguchiya et al., 2011). The underlying reason may be that the PVL toxin produced increases the survival advantage of CA strains. Furthermore, in this study, one strain was detected with coexisting $p v l, s e c$, and $t s s t-l$ genes. According to the case history data, this patient suffered from phlegmon in the left leg and presented with fever, dizziness, diarrhea, local abscesses, and myalgia. One strain was detected with the coexistence of PVL and SEC. This patient had a soft tissue infection on the foot and presented with local suppuration, incidental nausea, and vomiting. Both patients received vancomycin for anti-inflammation as well as incision and drainage, and good outcomes were achieved. Enterotoxin detection showed that the SEC carrying rate of CA-MSSA was 25\%. No SEH or ET carrying strain was detected. Positive toxin rates are presumably correlated with infection types and strain sources.

CA-MSSA has relatively low drug resistance (Guo et al., 2013), its genetic backgrounds are diverse (Mehndiratta and Bhalla, 2012), and it possesses clones identical to MRSA. Therefore, great attention should be paid to infection-causing MSSA in clinical practice, particularly PVL-producing strains, to prevent its prevalence and spread (Skov et al., 2012).

\section{REFERENCES}

Chen FJ, Hiramatsu K, Huang IW, Wang CH, et al. (2009). Panton-Valentine leukocidin (PVL)-positive methicillinsusceptible and resistant Staphylococcus aureus in Taiwan: identification of oxacillin-susceptible mecA-positive methicillin-resistant S. aureus. Diagn. Microbiol. Infect. Dis. 65: 351-357.

Chen H, Liu Y, Jiang X, Chen M, et al. (2010). Rapid change of methicillin-resistant Staphylococcus aureus clones in a Chinese tertiary care hospital over a 15-year period. Antimicrob. Agents Chemother. 54: 1842-1847.

Chen FJ, Siu LK, Lin JC, Wang CH, et al. (2012). Molecular typing and characterization of nasal carriage and communityonset infection methicillin-susceptible Staphylococcus aureus isolates in two Taiwan medical centers. BMC Infect. Dis. 12: 343.

Clinical and Laboratory Standards Institute (2010). Performance Standards for Antimicrobial Susceptibility Testing; Twentieth Informational Supplement. CLSI document M100-S20, CLSI, Wayne.

DeLeo FR, Otto M, Kreiswirth BN and Chambers HF (2010). Community-associated meticillin-resistant Staphylococcus aureus. Lancet 375: 1557-1568. 
Du J, Chen C, Ding B, Tu J, et al. (2011). Molecular characterization and antimicrobial susceptibility of nasal Staphylococcus aureus isolates from a Chinese medical college campus. PLoS One 6: e27328.

Escobar JA, Gómez IT, Murillo MJ, Castro BE, et al. (2012). Design of two molecular methodologies for the rapid identification of Colombian community-acquired methicillin-resistant Staphylococcus aureus isolates. Biomedica 32: 214-223.

Guo Y, Ramos RI, Cho JS, Donegan NP, et al. (2013). In vivo bioluminescence imaging to evaluate systemic and topical antibiotics against community-acquired methicillin-resistant Staphylococcus aureus-infected skin wounds in mice. Antimicrob. Agents Chemother. 57: 855-863.

Huang YC and Chen CJ (2011). Community-associated meticillin-resistant Staphylococcus aureus in children in Taiwan, 2000s. Int. J. Antimicrob. Agents 38: 2-8.

Kaltsas A, Guh A, Mediavilla JR, Varshney AK, et al. (2011). Frequency of panton-valentine leukocidin-producing methicillin-sensitive Staphylococcus strains in patients with complicated skin and soft tissue infection in Bronx, New York. J. Clin. Microbiol. 49: 2992-2995.

Kawaguchiya M, Urushibara N, Kuwahara O, Ito M, et al. (2011). Molecular characteristics of community-acquired methicillin-resistant Staphylococcus aureus in Hokkaido, northern main island of Japan: identification of sequence types 6 and 59 Panton-Valentine leucocidin-positive community-acquired methicillin-resistant Staphylococcus aureus. Microb. Drug Resist. 17: 241-250.

Khokhlova O, Tomita Y, Hung WC, Takano T, et al. (2012). Elderly infection in the community due to ST5/SCCmecII methicillin-resistant Staphylococcus aureus (the New York/Japan clone) in Japan: Panton-Valentine leukocidinnegative necrotizing pneumonia. J. Microbiol. Immunol. Infect. (Epub ahead of print)

Kini AR, Shetty V, Kumar AM, Shetty SM, et al. (2013). Community-associated, methicillin-susceptible, and methicillinresistant Staphylococcus aureus bone and joint infections in children: experience from India. J. Pediatr. Orthop. B. 22: $158-166$.

Lindsay JA and Holden MT (2006). Understanding the rise of the superbug: investigation of the evolution and genomic variation of Staphylococcus aureus. Funct. Integr Genomics 6: 186-201.

Liu Y, Wang H, Du N, Shen E, et al. (2009). Molecular evidence for spread of two major methicillin-resistant Staphylococcus aureus clones with a unique geographic distribution in Chinese hospitals. Antimicrob. Agents Chemother. 53: 512518.

Mehndiratta PL and Bhalla P (2012). Typing of methicillin resistant Staphylococcus aureus: a technical review. Indian J. Med. Microbiol. 30: 16-23.

Mithoe D, Rijnders MI, Roede BM, Stobberingh E, et al. (2012). Prevalence of community-associated meticillin-resistant Staphylococcus aureus and Panton-Valentine leucocidin-positive S. aureus in general practice patients with skin and soft tissue infections in the northern and southern regions of The Netherlands. Eur. J. Clin. Microbiol. Infect. Dis. 31: 349-356.

Pandian DG, Babu RK, Chaitra A, Anjali A, et al. (2011). Nine years' review on preseptal and orbital cellulitis and emergence of community-acquired methicillin-resistant Staphylococus aureus in a tertiary hospital in India. Indian J. Ophthalmol. 59: 431-435.

Pardo L, Vola M, Macedo-Viñas M, Machado V, et al. (2013). Community-associated methicillin-resistant Staphylococcus aureus in children treated in Uruguay. J. Infect. Dev. Ctries. 7: 10-16.

Rodríguez-Baño J, Angeles DM, Blas MA, Borraz C, et al. (2009). Clinical and molecular epidemiology of communityacquired, healthcare-associated and nosocomial methicillin-resistant Staphylococus aureus in Spain. Clin. Microbiol. Infect. 15: 1111-1118.

Schijffelen MJ, Boel CH, van Strijp JA and Fluit AC (2010). Whole genome analysis of a livestock-associated methicillinresistant Staphylococcus aureus ST398 isolate from a case of human endocarditis. BMC Genomics 11: 376.

Skov R, Christiansen K, Dancer SJ, Daum RS, et al. (2012). Update on the prevention and control of community-acquired meticillin-resistant Staphylococcus aureus (CA-MRSA). Int. J. Antimicrob. Agents 39: 193-200.

Smith TC and Pearson N (2011). The emergence of Staphylococcus aureus ST398. Vector Borne Zoonotic. Dis. 11: 327339.

Sun MT, Simon S, Warner M, Chehade M, et al. (2012). Methicillin-resistant Staphylococus aureus. Ophthalmology 119: 1719.

Wu D, Wang Q, Yang Y, Geng W, et al. (2010). Epidemiology and molecular characteristics of community-associated methicillin-resistant and methicillin-susceptible Staphylococcus aureus from skin/soft tissue infections in a children's hospital in Beijing, China. Diagn. Microbiol. Infect. Dis. 67: 1-8.

Zhao C, Liu Y, Zhao M, Liu Y, et al. (2012). Characterization of community acquired Staphylococcus aureus associated with skin and soft tissue infection in Beijing: high prevalence of PVL+ST398. PLoS One 7: e38577. 